\title{
Center for stroke disparities solutions community- based care transition interventions: study protocol of a randomized controlled trial
}

\author{
Penny H Feldman ${ }^{1 *}$, Margaret V McDonald ${ }^{2}$, Melissa A Trachtenberg ${ }^{2}$, Antoinette Schoenthaler ${ }^{3}$,
} Noreen Coyne ${ }^{2}$ and Jeanne Teresi ${ }^{4}$

\begin{abstract}
Background: Racial and ethnic disparities persist in stroke occurrence, recurrence, morbidity and mortality. Uncontrolled hypertension (HTN) is the most important modifiable risk factor for stroke risk. Home health care organizations care for many patients with uncontrolled HTN and history of stroke; however, recurrent stroke prevention has not been a home care priority. We are conducting a randomized controlled trial (RCT) to compare the effectiveness, relative to usual home care (UHC), of two Community Transitions Interventions (CTIs). The CTIs aim to reduce recurrent stroke risk among post-stroke patients via home-based transitional care focused on better HTN management.

Methods/Design: This 3-arm trial will randomly assign 495 black and Hispanic post-stroke home care patients with uncontrolled systolic blood pressure (SBP) to one of three arms: UHC, UHC complemented by nurse practitionerdelivered transitional care (UHC + NP) or UHC complemented by an NP plus health coach (UHC $+\mathrm{NP}+\mathrm{HC})$. Both intervention arms emphasize: 1) linking patients to continuous, responsive preventive and primary care, 2) increasing patients'/caregivers' ability to manage a culturally and individually tailored BP reduction plan, and 3) facilitating the patient's reintegration into the community after home health care discharge. The primary hypothesis is that both NP-only and NP + HC transitional care will be more effective than UHC alone in achieving a SBP reduction. The primary outcome is change in SPB at 3 and 12 months. The study also will examine cost-effectiveness, quality of life and moderators (for example, race/ethnicity) and mediators (for example, changes in health behaviors) that may affect treatment outcomes. All outcome data are collected by staff blinded to group assignment.
\end{abstract}

Discussion: This study targets care gaps affecting a particularly vulnerable black/Hispanic population characterized by persistent stroke disparities. It focuses on care transitions, a juncture when patients are particularly susceptible to adverse events. The CTI is innovative in adapting for stroke patients an established transitional care model shown to be effective for HF patients, pairing the professional NP with a HC, implementing a culturally tailored intervention, and placing primary emphasis on longer-term risk factor reduction and community reintegration rather than shorter-term transitional care outcomes.

Trial registration: ClinicalTrials.gov NCT01918891; Registered 5 August 2013.

Keywords: Care transitions, Home health, Stroke, Hypertension, Blood pressure, Health disparities, Trial design

\footnotetext{
* Correspondence: pfeldman@vnsny.org

${ }^{1}$ Center for Home Care Policy and Research, Visiting Nurse Service of New

York, 107 East 70th Street, 10021 New York, NY, USA

Full list of author information is available at the end of the article
} 


\section{Background}

Despite improvements in reducing stroke first-ever occurrence, mortality and recurrence over the past few decades [1-3], racial and ethnic disparities persist. Risk of having a first stroke for blacks is nearly twice as high as for whites, and blacks are more likely to die following a stroke than are whites [4]. The risk of stroke for Hispanics falls between that of whites and blacks [4]. A similar pattern is seen in recurrent stroke, with blacks and Hispanics having higher recurrence rates than whites [5-8]. Approximately, one quarter of the strokes that occur each year are in people who had a previous stroke [4]. Compared to first strokes, recurrent strokes are associated with higher mortality, greater disability, and greater health care costs $[9,10]$.

While reasons for racial and ethnic disparities in stroke and stroke recurrence are complex, numerous studies have demonstrated that blacks and Hispanics have disproportionately higher rates of stroke risk factors than whites, including uncontrolled hypertension (HTN), poorer diabetes control, and higher rates of hyperlipidemia $[11,12]$. HTN is a particularly important factor as it has been found repeatedly to be a predictor of stroke and stroke recurrence in the black and Hispanic populations [11-15] and has been identified as the single most important modifiable stroke risk factor [14,16,17]. Multiple studies and clinical guidelines have been published indicating the benefits of HTN control on reducing stroke risk [18-20]. Authors of a review of prospective cohort studies and a meta-analysis of over 40 randomized controlled trials found that in both sets of studies each $10 \mathrm{mmHg}$ lower systolic blood pressure (SBP) was associated with a one third reduction in stroke risk [19].

Despite the benefit of HTN control, a large treatment gap exists between the medical and behavioral regimens recommended for recurrent stroke prevention and actual post-stroke care received by patients [21]. The American Heart Association and American Stroke Association (AHA/ASA) jointly issued a statement in 2011, acknowledging racial and ethnic disparities in different patients' stroke experiences, including access to and quality of care [22]. Our study seeks to address disparities and service gaps by identifying and intervening with a particularly vulnerable population - black and Hispanic patients who have a history of stroke, currently have uncontrolled HTN and are patients transitioning in and out of post-acute home health care services. Data from the National Home and Hospice Survey, which is conducted by the Centers for Disease Control, indicate that $7 \%$ of home care patients have a history of cardiovascular disease and $41 \%$ have HTN [23]. Each day there are approximately 1.5 million patients enrolled in home health care [23].

Home care not only constitutes an unexplored environment to intervene to prevent stroke recurrence, it also presents an opportunity to do so in the context of care transitions, a time when patients are particularly susceptible to adverse events [24,25]. This Community Transitions Intervention (CTI) study aims to assess the comparative effectiveness, relative to usual home care (UHC), of adding a nurse practitioner (NP)-delivered transitional care intervention or a transitional care intervention delivered by an NP + health coach $(\mathrm{HC})$. Clinical trials of NP-delivered transitional care interventions have shown them to be effective in improving outcomes of heart failure (HF) patients [24]. Trials of community health worker and coaching interventions have shown significant potential to improve blood pressure (BP) control [26-29]. However, NP transitional care alone or combined with $\mathrm{HC}$ support has never been tested as a vehicle for risk reduction among post-stroke patients.

Both intervention arms have a three-fold focus: 1) linking patients to continuous, rapidly responsive preventive and primary care, 2) increasing patients'/caregivers' ability to manage a culturally and individually tailored BP reduction plan, and 3) facilitating the patient's reintegration into the community after home health care discharge. The CTI is significant in that it targets care gaps affecting a particularly vulnerable population characterized by persistent stroke disparities (blacks/Hispanics). It is innovative in that it adapts for stroke patients established transitional care models shown to be effective for HF patients [26,30], pairs the professional NP with a community $\mathrm{HC}$ and gives emphasis to longer-term risk factor reduction and community reintegration rather than short-term transitional care outcomes.

\section{Methods/Design}

The study is designed as a three-arm RCT so that we can test individually the comparative effectiveness of adding: a) just an NP CTI, or b) an NP + HC CTI to routine care -that is UHC. The primary hypothesis is that either intervention will be more effective in reducing blood pressure than the UHC condition. There is no hypothesis regarding the relative merits of one of the intervention arms over the other. Thus, the study is powered to compare the effects of each intervention (NP and NP $+\mathrm{HC}$ ) to UHC. However, in sensitivity power calculations for non-linear change models, scenarios with different effect sizes associated with each intervention will be examined.

\section{Setting: study site}

The study is being conducted in the post-acute care division of a large, urban, nonprofit, Medicare-certified home health organization. The majority of post-acute care stroke patients are admitted to home care immediately after a hospital discharge; others come through community referrals (primarily physicians). All patients in the study (regardless of study arm) will receive UHC (see Figure 1). The study was approved by the Visiting Nurse Service of 


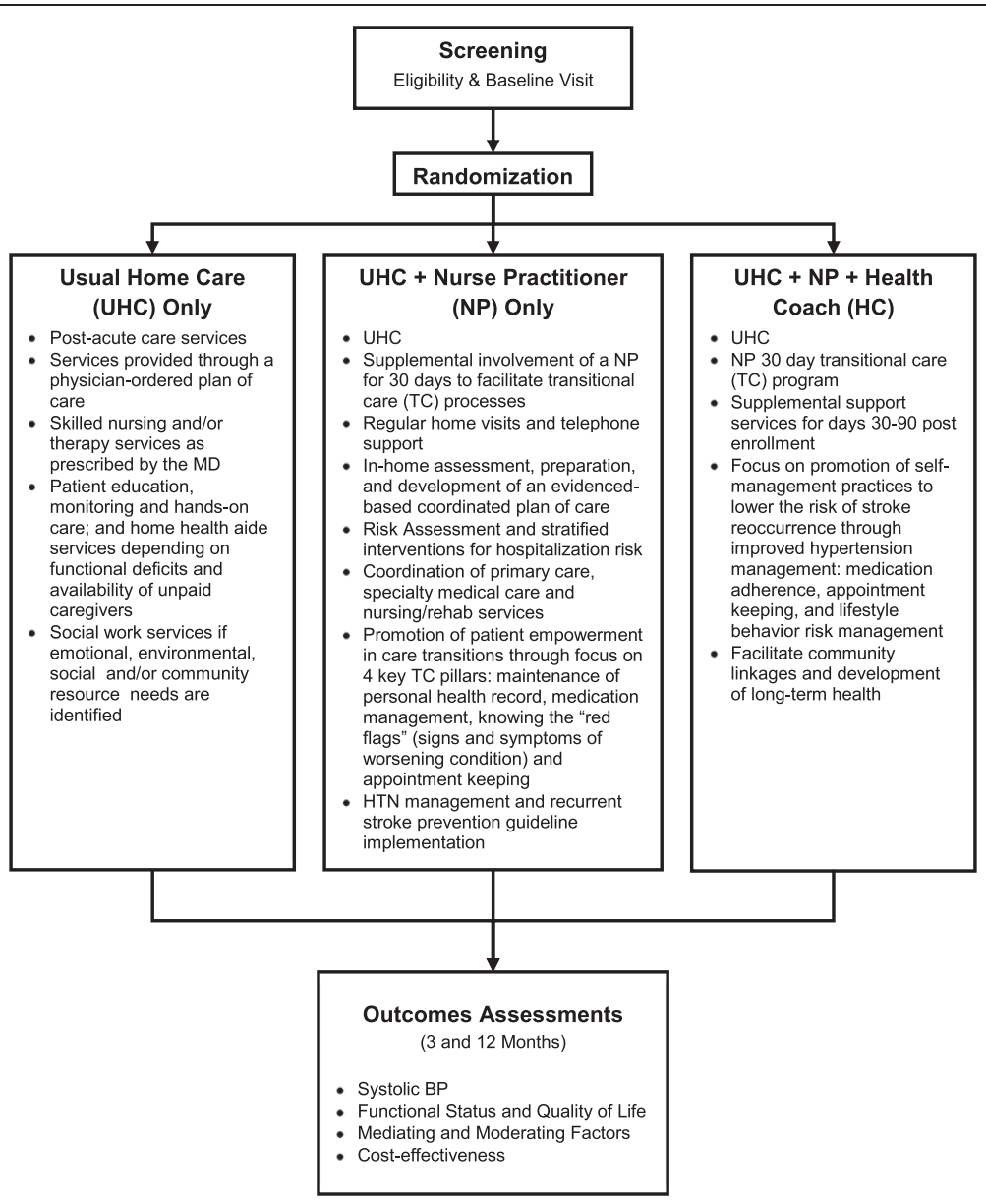

Figure 1 Community Transitions Intervention study design.

New York's (VNSNY's) institutional review board (reference \#I12-004).

\section{Control condition: usual home care}

UHC consists of: a physician-ordered plan of care; skilled nursing and/or therapy services as prescribed by the medical doctor; patient education, monitoring and hands-on care; home health aide services depending on functional deficits and availability of unpaid caregivers; and social work services if emotional, environmental, social and/or community resource needs are identified. All nurses and therapists use tablet computers, electronic messaging and an electronic health record (EHR); and have access to a decision support system. The decision support system includes a discretionary module on care of stroke patients that outlines information to teach the patient/ caregiver (for example, manifestations/causes/contributing factors to stroke, behaviors to manage a stroke, and symptoms/complications to seek emergency care). The module also gives nurses information on helping patients manage motor/sensory/cognitive deficits. The module emphasizes stroke recovery/rehabilitation rather than secondary prevention.

\section{Interventions}

\section{Nurse Practitioner (NP) Arm}

The study's NPs are employed by a professional practice group affiliated with the parent home health organization. The practice group provides primary and specialty medical services and care coordination and can bill third parties. The intervention, which complements UHC, builds on an existing 30-day transitional care program established in the practice group. Program components are modeled after two evidence-based models: 1) the Transitional Care Model (TCM) developed and validated for HF patients [26], which uses an advanced practice nurse for intervention activities, and 2) the Four Pillars of the 30-day Care Transitions Intervention ${ }^{\circledR}$ (CTI) [30], which emphasizes patient self-management. Both models have demonstrated effectiveness in reducing hospital readmissions [25,31]. Because the intervention commences only after a patient is admitted to home care, we have 
omitted the element that calls for visiting the patient in the hospital before discharge. This element has been omitted in other tests of the TCM [32]. We also have modified the traditional TCM in at least two other ways: 1) to allow for the inclusion of at-risk eligible patients referred to home care from community physicians, and 2) to increase the emphasis on stroke recurrence prevention - specifically on reducing SBP. We incorporated and combined the goal of improved transitional care with the goal of effective chronic disease self-management. The program length of 30 days was chosen because it is the allowable Medicare reimbursable timeframe for transitional care services and thus should contribute to the sustainability of the model after the grant period ends.

With intervention patients the NP is responsible for: 1) conducting a comprehensive health assessment; 2 ) establishing linkages with the patient's physicians (specialists and primary care) and UHC nurse; 3) working with the physicians to specify recommended medication and behavioral regimens; 4) coordinating with the patient and the patient's caregivers to formulate patient goals; 5) tailoring the treatment plan to the patient's preferences and circumstances; and 6) providing collaborative problem solving and self-management support to the patient and caregivers. The NP patient-contact protocol includes: 3 in-home visits, 3 patient-caregiver telephone calls, and a varied amount of inter-professional collaboration calls over the 30-day intervention period. Between the in-home visits, the patient-caregiver calls provide the opportunity for the NP to review with patients/caregivers their medical appointments, test results and medication issues and to reinforce progress with lifestyle management. The NP also may attend a joint visit to the patient's primary care physician for patients who are having difficulty with their treatment plan. Additional resources that the NP provides and discusses with the patient/caregiver are a transitional care booklet that includes worksheets to be used to develop a personal health record and an (AHA/ ASA) packet 'Understanding and Controlling your High Blood Pressure' educational guide. These guides are provided in English or Spanish as preferred by the patient.

\section{$N P+$ health coach $(H C)$ Arm}

This arm, like the NP-only arm, complements UHC. It is designed so that all patients receive the intensive 30-day NP intervention as described above plus an additional 60 days of collaboration and self-management support from a specially trained $\mathrm{HC}$. The $\mathrm{HC}$ joins the NP at the third patient intervention visit and subsequently provides 3 in-home visits and 3 telephone calls for $\mathrm{HC}$ /patientcaregiver collaboration. The planned schedule of NP/HC contacts is outlined in Table 1.

Home health aides and other community members who have similar racial and ethnic backgrounds as the
Table 1 Intervention staff-patient encounter schedule

\begin{tabular}{lll}
\hline Week & NP & HC \\
\hline 1 & Visit & \\
& Call & \\
2 & Visit & \\
3 & Call & Visit \\
4 & Call & \\
& Visit & Call \\
5 & & \\
7 & & Visit \\
8 & & Call \\
9 & & \\
10 & & Call \\
11 & & \\
12 & & Visit \\
\hline
\end{tabular}

Notes: $\mathrm{HC}=$ health coach; $\mathrm{NP}=$ nurse practitioner.

target patient population have been engaged and trained as community health coaches for this study. The training curriculum was based on the principles of adult learning theory, the core competencies identified by the National Community Health Advisor study [33], and the Training Curriculum for Health Coaches developed by Bodenheimer at UCSF Center for Excellence in Primary Care [34]. The coaches were trained to use motivational interviewing strategies (for example, setting the agenda, reflective listening, building motivation for change, goal setting) [35] to facilitate relationship building, assist with problem-solving, and to promote behavior change. Training was led by one of the co-investigators (AS), who is a member of the Motivational Interviewing Network of Trainers (MINT).

The key elements of the HC role are: 1) communication with patients and their informal caregivers to promote recurrent stroke prevention awareness, education, and understanding of risk factors and recommended medical and lifestyle regimens; 2) collaborative interactions to improve self-management; and 3) navigation and networking to facilitate patients' social and physical integration into the community for lasting change. The $\mathrm{HC}$ uses the AHA/ASA educational guide distributed by the NP, as noted above, to support his or her efforts in working with the patient on setting behavior change goals.

\section{Intervention fidelity monitoring protocol}

Monitoring activities are outlined below:

1. Review NP adherence to intervention protocol Primary monitoring

a. \#/\% of 6 encounters planned; track \#/\% of 3 in-home visits and $\# / \%$ of 3 telephone calls 
b. \# of encounters above the 6 planned

c. \#/\% of cases in which HTN management was addressed

d. \#/\% of cases joint NP-HC visit was completed, when appropriate

Supplemental monitoring

e. \#/\% of patients who have a joint NP-MD visit

f. \# of collaborative-coordination calls per patient

2. Review health coach adherence to intervention protocol

Primary monitoring

a. \#/\% of 6 encounters planned; track \#/\% of 3 in-home visits and \#/\% of 3 telephone calls

b. \# of encounters above the 6 planned

c. \#/\% of cases in which at least $1 \mathrm{HTN}$ self-management goal was established

d. Completion rate of requested audio-recordings

e. \#/\% of audio-recordings in which motivational interviewing principles were demonstrated

Supplemental monitoring

f. \#/\% of patients who receive a community resource referral

Abbreviations; $\mathrm{HC}=$ health coach; $\mathrm{HTN}=$ hypertension; $\mathrm{MD}=$ doctor of medicine, $\mathrm{NP}=$ nurse practitioner.

$\mathrm{NP}$ and $\mathrm{HC}$ intervention staff enter information on each of their patient encounters in the EHR of the transitional care program. Patient-level and aggregate reports, generated for regular review by the principal investigator, the project manager and the field coordinator, include data on the number and types of encounters by each discipline, time of each encounter, and number of collaborativecoordination calls. Data about HTN management interventions and goal setting/achievement is also being extracted, tracked, and monitored. NPs are involved in regular case reviews. The health coaches are audio-recording encounters with $20 \%$ of their patients. These sessions are being evaluated using the Behavior Change Counseling Index (BECCI) [36]. The BECCI is an instrument designed for trainers to score practitioners' use of behavior change counseling strategies in their patient encounters. Coaches receive regular feedback on their intervention activities by the project manager and two MINT trainers.

\section{Eligibility and inclusion/exclusion criteria}

A total of 495 black and Hispanic patients ( $\geq 21$ years of age) who have had a first time or recurrent stroke (ischemic or hemorrhagic) or transient ischemic attack (TIA) and have uncontrolled SBP will be recruited: 165 randomized to each group. To be eligible a patient also needs to be English or Spanish speaking and have a telephone to use for intervention encounters. Patients are excluded if they have a clinical condition that may require specialized HTN management (for example, end stage renal disease, severe HF). Additional inclusion/exclusion criteria are listed in Table 2.

\section{Procedures \\ Screening}

Initial identification is through a review of EHR data for new admissions into the organization's post-acute care program. Electronic records were used to initially identify potential patients using a variety of International Classification of Diseases, version 9 (ICD-9) codes for post-stroke care (with the vast majority of patients coming in with a code of 438.xx - late effects of cerebrovascular disease). Confirmation of the history of stroke or TIA was completed through patient self-report. After patients are enrolled, they are asked to sign a release for their hospital discharge summaries so the investigator group can retrieve additional information on their most recent stroke. Confirmation of additional eligibility criteria requires that the patient pass: 1) a telephone eligibility screen, 2) an initial in-home BP check, and 3) a confirmatory BP check within 7 days of the initial check. These three steps are conducted by specially trained study interviewers who receive extensive didactic, role play, and field training prior to independent deployment.

On the first BP screening visit, three readings are taken simultaneously on both arms using a validated, automated oscillometric BP device (Microlife Watch BP, Golden, CO, USA) and an average is provided. If a patient's SBP is $140 \mathrm{mmHg}$ or greater on either arm, the patient is scheduled for a follow up in-home BP screening

\section{Table 2 Study participant eligibility criteria}

\begin{tabular}{|c|c|}
\hline Inclusion criteria & Exclusion criteria \\
\hline $\begin{array}{l}\text { - Average screening systolic } \\
\mathrm{BP} \geq 140 \mathrm{mmHg} \text { on } 2 \text { screening } \\
\text { visits within } 7 \text { days of each other }\end{array}$ & $\begin{array}{l}\text { - Average screening systolic BP } \geq \\
200 \mathrm{mmHg} \text { or average diastolic } \\
\mathrm{BP} \geq 120 \mathrm{mmHg} \text { (on } 3 \text { consecutive } \\
\text { visits) }\end{array}$ \\
\hline \multirow{2}{*}{$\begin{array}{l}\text { - Recently admitted to the home } \\
\text { care post-acute care program }\end{array}$} & - Dialysis ${ }^{a}$ \\
\hline & - End stage renal disease ${ }^{a}$ \\
\hline - 21 years of age or older & - Kidney transplant ${ }^{a}$ \\
\hline - Black and/or Hispanic & - Severe heart failure ${ }^{a}$ \\
\hline - Speaks English or Spanish & \multirow{2}{*}{$\begin{array}{l}\text { - Significant cognitive impairment. } \\
\text { Unable to provide informed } \\
\text { consent, accurate self-report, } \\
\text { and/or unable to participate } \\
\text { effectively in intervention }\end{array}$} \\
\hline $\begin{array}{l}\text { - History of stroke (ischemic } \\
\text { or hemorrhagic) or transient } \\
\text { ischemic attack }\end{array}$ & \\
\hline - HTN diagnosis & \multirow{3}{*}{$\begin{array}{l}\text { - Patients with upper arm } \\
\text { circumference } \geq 38 \mathrm{~cm} \text {. At this } \\
\text { dimension our blood pressure } \\
\text { cuffs become inaccurate }\end{array}$} \\
\hline $\begin{array}{l}\text { - Resides in the study } \\
\text { catchment area }\end{array}$ & \\
\hline $\begin{array}{l}\text { - Has a telephone (in order } \\
\text { to participate in intervention } \\
\text { phone sessions) }\end{array}$ & \\
\hline
\end{tabular}

Notes: BP = blood pressure; HTN = hypertension.

${ }^{a}$ Conditions require very specialized care of HTN, including different medications and dietary/physical activity recommendations. 
within 7 days. On the second visit, $\mathrm{BP}$ is re-measured three times on the arm that had the higher SBP reading (the 'dominant arm'). If the average SBP is again $\geq 140 \mathrm{mmHg}$, the level that meets our study criteria, the interviewer proceeds with recruitment, the informed consent process. Patients with a systolic $\mathrm{BP} \geq 200 \mathrm{mmHg}$ or diastolic $\mathrm{BP}$ of $\geq$ $120 \mathrm{mmHg}$ at the screening visits are directed to receive immediate medical attention. If the patient is willing, an additional BP screening visit will be scheduled for at least 48 hours after the critical BP screen visit to determine potential eligibility. If the patient's BP continues to be critical, they will be advised to seek immediate medical attention and will not be enrolled in the study.

\section{Informed consent}

If the patient has a SBP of $140 \mathrm{mmHg}$ or greater at both screenings then the interviewer proceeds with the informed consent process. The interviewer provides the patient with the hardcopy consent and among other things describes the three potential study groups, the content of the interventions, the baseline and follow up interview schedule, the sharing of information with others on the study team and that involvement is voluntary. The interviewer confirms that the patient understands what they are consenting to, and if the patient agrees two consents are signed: one for study files and one for the patient. Informed consent is obtained from each participant.

\section{Baseline assessment}

Once a consent form is signed, the interviewer proceeds directly with the baseline assessment. The assessment is a structured interview, largely made of up self-report measures. (See Measurements section below).

\section{Randomization and blinding}

Following the baseline interview, subjects are randomized to one of three arms. Upon randomization to one of the intervention arms, the UHC nurse who is designated as the main nurse organizing and providing routine home health visits to the patient is sent a secure Email message indicating the patient's involvement in the CTI. The Email outlines the objectives of the program and provides information on how to contact the transitional care NP involved in the case. As necessary/appropriate, the NP contacts the UHC nurse, just as the NP contacts the physician or any other health care provider of the patient as medically necessary. Throughout the course of the study, field interviewers collecting baseline and outcome data are blinded to the patient assignment group.

\section{Anticipated study characteristics}

The gender distribution is expected to reflect the home care population with the targeted clinical conditions. Because the goal of study is to determine whether the interventions will improve blood pressure control in racial minorities after stroke, the participants in this study will be self-identified as black or Hispanic. Based on available organization data and prior studies, the enrolled sample is expected to be $65 \%$ black $/ 35 \%$ Hispanic; $40 \%$ male $/ 60 \%$ female; with a mean age of 67 years.

\section{Measurements \\ Data sources}

To achieve our specific aims, we will make use of data from: 1) patient assessments and interviews conducted during the initial phone screen, at baseline (enrollment in the study), and 3 and 12 months post-study enrollment; and 2) intervention cost data collected especially for this study. Information to be obtained is summarized in Table 3. Additionally, patient-level clinical and functional assessment data derived from OASIS, the nationally mandated home care Outcomes Assessment and Information Set will be used to assess potential differences in those participating in the study and those who declined. This will be used for discussion of the generalizability of our findings.

\section{Primary outcome: change in systolic blood pressure}

The primary outcome is change in SBP from baseline to 3 and 12 months. As with the baseline interview, patients are assessed with a validated, automated oscillometric BP device (Microlife Watch BP, Golden, CO, USA). The average of the BP measurements from the two enrollment screening visits will be used for comparative analysis to the follow up measurements. At the 3- and 12-month follow up evaluations, the BP will be measured by interviewers, blinded to group assignment, 3 times on the dominant arm identified at baseline and the average will be used for analysis.

\section{Secondary outcomes: cost-effectiveness, patient function,} and quality of life

Costs will focus on direct costs including costs of the interventions, home care utilization, hospital and emergency department use, outpatient visits and medication regimens. Patient function and health-related quality of life will be assessed with a modified self-report Barthel Index [37] and the EuroQol [38]. See Table 3.

\section{Exploratory outcomes: moderators and mediators}

Data are being collected so that moderators and mediators that may affect treatment outcomes can be explored. Moderators include race and ethnicity (that is black/Hispanic) differences and baseline HTN severity (for example, Stage 1 (SBP $=140$ to $159 \mathrm{mmHg}$ ) versus Stage 2 (SBP $\geq 160 \mathrm{mmHg})$ ). Mediators include changes in health behaviors (for example, diet, physical activity, weight loss, medication adherence) and antihypertensive medication intensification. 
Table 3 Information to be collected and used in analysis

Baseline 3 Months 12 Months

\section{Patient Characteristics}

Basic sociodemographics: age, sex, race, ethnicity, preferred language to use, living situation, education, health literacy

Marital status, employment status, income

Acculturation: place of birth, parent and grandparent place of birth, length of time in US

Executive Cognitive Function (FAB)

Stroke, HTN and hospitalization history; co-morbidities (modified Charlson)

Clinical and functional outcomes

\section{Primary outcome}

Blood pressure measurement

\section{Secondary outcomes}

Cost-effectiveness

Health-related quality of life (EuroQol)

ADL/Functional Status (modified Barthel/Rankin for patient self-report)

Physical function (PROMIS ${ }^{\oplus}$ )

New cardiovascular events

\section{Exploratory outcome mediators}

Knowledge of HTN management

Stroke literacy questions (Willey)

Adherence to regimen (Morisky)

Beliefs about medications (BMQ)

Linkage to PCP

Chronic condition management (PACIC)

General self-efficacy scale (Lorig)

Lifestyle management: diet/sodium intake (modified CATCH), physical activity (IPAQ), tobacco, alcohol and drug use, body mass index

Depression (PROMIS $\left.{ }^{\oplus}\right)$

Medication profile

\section{Organizational and system costs}

Intervention costs: nurse practitioner and health coach time, patient educational material

Home care-related costs: nurse, therapy, social work, and aide home care visits

Overall medical costs: nights in hospital, ED visits, physician visits, medications

Baseline

onths

12 Months

$X$

$X$

$\mathrm{X}$

$X$

$X$

$X$

$x \quad x \quad x$

$x \quad x \quad x$

$x$

Notes: Barthel/Rankin [37,53]; FAB = Frontal Assessment Battery [54]; Charlson, modified self-report [55,56]; EuroQol [38]; PROMIS ${ }^{\circledR}$ [57]; Willey [58]; Morisky, 8-item version [59]; BMQ [60], PACIC = Patient Assessment of Chronic Illness Care, modified subscales [61]; Lorig [62]; CATCH [63]; IPAQ = International Physical Activity Questionnaire $[64,65] ; P^{2}$ ROMIS ${ }^{\oplus}$ depression [66]; $E D=$ emergency department; $A D L=$ activities of daily living; $P C P=$ primary care provider; $H T N=$ hypertension.

\section{Statistical analysis plan}

\section{Data analyses for primary outcome: SBP}

The main hypothesis is that those assigned to the intervention groups will, on average, exhibit greater 3-month and 12-month decreases in SBP than those assigned to the usual care condition. The primary proposed analyses will use mixed random effects models, and a full information maximum likelihood (FIML) approach, with sensitivity analyses using generalized estimating equation (GEE) regression models. The change from pre- to post-treatment values of SBP will be modeled as functions of time, treatment and the interaction of time and treatment. The general longitudinal mixed effects model, using SAS PROC MIXED (SAS, Cary, NC, USA), will be used to model serial correlations and group heterogeneity in residual variances if needed. The intent-to-treat (ITT) analyses will permit all individuals with at least one observation to be included. If a non-linear pattern of change is observed, non-linear models will be used in sensitivity analyses.

Prior to analyses, baseline values of all variables will be examined in order to determine if any covariates require modeling due to imbalance among groups. Examination of baseline differences on key variables between completers 
and those lost to follow up will be conducted to inform about the nature of the missing data.

\section{Power for SBP rate of change}

Power was calculated, examining the rate of change, including all three waves in the analyses. The following assumptions were used: $\mathrm{R}=0.90$ (reliability); pooled $\sigma=20.75 ; \delta=5,6,7$ (SBP point reduction per year in the intervention groups relative to the UHC group reduction); $\mathrm{d}=\delta / \sigma$ (where $\delta$ is point reduction per year and $\mathrm{d}$ is Cohen's $d$ for the rate of change); $\rho=0.6$ is the average correlation between baseline and follow up assessments; $\mathrm{Tn}=3$ time points (baseline, 3 month and 12 month). The formulas from Diggle, Liang and Zeger (1994) were used [39]: the power calculation demonstrates that under ITT, we can detect a rate of reduction in SBP equivalent to $5.40 \mathrm{mmHg}$ which translates to relatively small effect sizes using Cohen's d. It is also noted that the assumed standard deviation was conservatively posited to be quite large (pooled estimate of around 21). If a smaller standard deviation is observed, power will be greater. Thus, a sample size of 165 per group was proposed.

For the non-linear, longitudinal change sensitivity analyses [40], the assumptions were the same as above, and an endpoint reduction in SBP of 6 points was posited. Assuming a non-linear decline function and differential treatment effects, power was greater than 0.80 to detect small effects (for example, a 0.5 point reduction) in one intervention group at 3 months and medium to large effects ( 2.5 to 5 point reduction in SBP) in the second group at 3 months, with a net reduction of 6 points by study end in both groups.

\section{Discussion}

The cumulative 5-year risk of having a recurrent stroke after a person's first-ever stroke is over $30 \%$, and the 30 day fatality risk associated with secondary strokes has been reported to be 40 to $50 \%$, which is significantly higher than first stroke fatality rates [41,42]. Blacks and Hispanics have higher risk of recurrence than whites $[5,7,8]$. A contributor to this ongoing risk of stroke survivors is ineffective recurrent stroke preventive interventions. Our interventions are designed to specifically address HTN, a major risk factor for strokes and recurrent strokes, and are built on NP and HC models found to be successful in addressing other clinical conditions and established in other care settings. The NPs are positioned to complement UHC services by assessing and addressing hospitalization risk factors that are common during transitional care periods, while also providing a specific HTN management intervention geared toward preventing stroke recurrence. Both the NP and NP + $\mathrm{HC}$ interventions expand on the usual transitional care focus, which is on hospital discharges and preventing rehospitalization, by focusing on longer-term stroke risk prevention, community provider connections to facilitate long-term chronic care management, and reintegration into the community.

Our interventions were informed by models that showed promise in other settings. Focus groups and individual interviews were also conducted with black and Hispanic post-stroke patients who had received home care and their caregivers to further inform our intervention approach along with our enrollment efforts. During these focus groups, post-stroke patients expressed fear of research and lack of trust in researchers. They suggested we might counter these fears by making sure that potential patients are able to get a very clear understanding of the study and their expected involvement. Operationally, this led us to simplify the language used in describing the study: for example, the randomization process (including being clear about the possibility of being placed in the usual care group) and the intent of the interventions. We also built more time into the recruitment calls and in-person consenting process so that the patients are free to ask all their questions. In addition, field staff were trained in the 'teach back' method - asking patients to describe in their own words what the initiative was about and what their commitment was in order to determine areas that needed further clarification. Lastly, we incorporated the suggestion of focus group members that we stress the useful knowledge gained through research and how patients are the ones with the important information.

The intervention approach already stressed patientcenteredness in goal setting, but focus group findings led us to enhance staff training to further address the cultural issues that came up at our meetings. For example, to address the issue of trust or rather distrust in the medical care systems, we instituted staff discussions and practice sessions on how to maintain trust (for example, reflective listening, following up with community referrals when requested, addressing other clinical conditions if priority to the patient). Some of our focus group participants also expressed a sense of inevitability to their stroke experience and the possibility of having another stroke. These feelings seem to have been influenced partly by the view that chronic disease, hypertension, and diabetes are 'passed down' in the family. During our staff training, we discussed the implications of these beliefs (for example, how they may impact a person's selfmanagement behavior), how to identify these beliefs, and how to address them (for example, additional education). We also were guided by our participants to engage family and others who provided the patient's primary social support if the patient indicated that this was desirable, to take the time to address medication administration and side effect questions and to explore patients' attitudes and beliefs about medications - elements all integrated into 
the intervention approach. Additional direction for intervention staff on how to recognize depression and isolation risk was provided in the training as these were areas that our focus group informants felt could hinder adherence to recommended management guidelines and their recovery.

Two significant issues related to BP criteria for patient eligibility arose shortly before and after enrollment began, and both had potentially significant operational implications. A few weeks before launch the Program Advisory Committee for the Center for Stroke Disparities Solutions (CSDS) [43], parent of our study, advised the team to modify the BP eligibility screening process. Initially, the protocol was designed to determine patient eligibility based on a single face-to-face encounter in which the average of 3 SBP readings needed to be $\geq 140 \mathrm{mmHg}$ after the patient passed other eligibility screening questions described above. The revised protocol calls for 2 face-to-face interviews in which $3 \mathrm{BP}$ readings are taken and need to result in an average $\mathrm{SBP}$ of $\geq 140 \mathrm{mmHg}$ in both interviews. Currently, there is no widely accepted scientific standard for enrolling patients in BP studies; some use historical data in medical records to identify potential study subjects $[44,45]$, some enroll patients if they simply have a HTN diagnosis [46], some allow enrollment of those in pre-HTN stage [47], others adopt a higher SBP threshold of $\geq 150 \mathrm{mmHg}$ at the time of enrollment [48] and some include patients with single BP measurements in the uncontrolled HTN range $[49,50]$. The intent of the more stringent screen employed by our trial is to increase the likelihood of enrollment of the intended target population - those with uncontrolled HTN. Adjustments to our workflow and budget were implemented, although it was unclear how many people would drop out after the second BP screening visit. Data from this revised screening process will help to inform future studies. As of 5 September 2014, of the 97 patients who passed the first BP screen, 65 (67\%) met the SBP threshold at the second $\mathrm{BP}$ screen.

The second BP threshold issue that came up shortly after enrollment began derived from publication of the 2014 Evidence-Based Guideline for the Management of High Blood Pressure in Adults [51]. One of the 9 recommendations made is to treat to a goal of $\mathrm{SBP}<150 \mathrm{mmHg}$ for the general population aged 60 years and over, a departure from the prior guideline [52], which recommended the SBP goal of $<140 \mathrm{mmHg}$ for all patients. None of the new recommendations addressed the goal SBP for post-stroke patients. We expect that around $60 \%$ of the patients enrolled in our study will be aged 60 years or over. Since there is a body of evidence showing the benefit of lower SBP for stroke risk reduction, and after a discussion with HTN specialists at the Center for Stroke Disparities Solutions, we did not alter our intervention approach to increase our SBP target to $<150 \mathrm{mmHg}$. However, there is some concern that primary care providers overseeing the long-term care of these patients may be influenced by these published guidelines and decline to more aggressively treat patients with SBP levels between 140 and $149 \mathrm{mmHg}$.

Results of this trial will provide important information on the design of interventions to address treatment gaps and disparities in recurrent stroke prevention for vulnerable black and Hispanic populations. The trial focuses on care transitions, a juncture when patients are particularly susceptible to adverse events. The interventions are innovative in adapting for stroke patients an established transitional care model shown to be effective for HF patients, pairing the professional $\mathrm{NP}$ with a $\mathrm{HC}$, tailoring the interventions for black and Hispanic patients respectively, and placing primary emphasis on longer-term risk factor reduction and community reintegration rather than shorter-term transitional care outcomes.

\section{Trial status}

Patient enrollment began in January 2014. Enrollment period is expected to be 30 months.

\section{Abbreviations}

ADL: activities of daily living; AHA: American Heart Association; ASA: American Stroke Association; BECCl: Behavior Change Counseling Index; BP: blood pressure; BMQ: beliefs about medications; CSDS: Center for Stroke Disparities Solutions; CTI: Care Transitions Intervention ${ }^{\oplus}$;

CTIs: Community Transitions Interventions; EHR: electronic health record; EuroQol: health-related quality of life; FAB: Frontal Assessment Battery; FIML: full information maximum likelihood; GEE: generalized estimating equation; HC: health coach; HF: heart failure; HTN: hypertension;

ICD-9: International Classification of Diseases, version 9; IPAQ: International Physical Activity Questionnaire; ITT: intent-to-treat; MINT: Motivational Interviewing Network of Trainers; NP: nurse practitioner; PCP: primary care provider; OASIS: Outcomes Assessment and Information Set; PACIC: Patient Assessment of Chronic Illness Care; RCT: randomized controlled trial; SBP: systolic blood pressure; TCM: Transitional Care Model; TIA: transient ischemic attack; UHC: usual home care; UHC + NP: usual home care complemented by a nurse practitioner; UHC + NP + HC: usual home care complemented by a nurse practitioner plus health coach; VNSNY: Visiting Nurse Service of New York.

\section{Competing interests}

The authors declare that they have no competing interests.

\section{Authors' contributions}

All authors read and approved the final version of the manuscript. PHF conceived of the study, led the development of its design and of this manuscript. MVM contributed to the conception and design of the study and co-authored this manuscript. MT contributed to the development of the enrollment and data collection protocol, and participated in the survey development along with providing a critical review of this manuscript. AS trained the NP and $\mathrm{HC}$ staff in patient-centered counseling and motivational interviewing techniques as well as monitoring the ongoing treatment fidelity of the HC's skills along with providing a critical review of this manuscript. NC participated in the study implementation and project planning, including development of the health coach curriculum and documentation system along with providing a critical review of this manuscript. JT was responsible for the statistical analyses plan along with providing a critical review of this manuscript.

\section{Acknowledgements}

The research protocol described in this publication was supported by the National Institute of Neurological Disorders and Stroke of the National Institutes of Health under Award Number U54NS081765. The content is 
solely the responsibility of the authors and does not necessarily represent the official views of the National Institutes of Health.

\section{Funding}

National Institute for Neurological Disorders and Stroke.

\section{Author details}

${ }^{1}$ Center for Home Care Policy and Research, Visiting Nurse Service of New York, 107 East 70th Street, 10021 New York, NY, USA. ${ }^{2}$ Center for Home Care Policy and Research, Visiting Nurse Service of New York, 5 Penn Plaza, 12th floor, 10001 New York, NY, USA. ${ }^{3}$ Center for Healthful Behavior Change, Department of Population Health, NYU School of Medicine, 227 East 30th Street, 634, 10016 New York, NY, USA. ${ }^{4}$ Columbia University Stroud Center and New York State Psychiatric Institute, Research Division, Hebrew Home at Riverdale, 5901 Palisade Avenue, 10471 Bronx, NY, USA.

\section{Received: 12 September 2014 Accepted: 5 January 2015}

Published online: 27 January 2015

\section{References}

1. Towfighi A, Saver JL. Stroke declines from third to fourth leading cause of death in the United States: historical perspective and challenges ahead. Stroke. 2011:42:2351-5

2. Hong K-S, Yegiaian S, Lee M, Lee J, Saver JL. Declining stroke and vascular event recurrence rates in secondary prevention trials over the past 50 years and consequences for current trial design. Circulation. 2011;123:2111-9.

3. Lackland DT, Roccella EJ, Deutsch AF, Fornage M, George MG, Howard G, et al. Factors influencing the decline in stroke mortality: a statement from the American Heart Association/American Stroke Association. Stroke. 2014:45:315-53.

4. Go AS, Mozaffarian D, Roger VL, Benjamin EJ, Berry JD, Blaha MJ, et al. Executive summary: heart disease and stroke statistics - 2014 update: a report from the American Heart Association. Circulation. 2014;129:399-410.

5. Feng W, Hendry RM, Adams RJ. Risk of recurrent stroke, myocardial infarction, or death in hospitalized stroke patients. Neurology. 2010;74:588-93.

6. Sheinart KF, Tuhrim S, Horowitz DR, Weinberger J, Goldman M, Godbold JH. Stroke recurrence is more frequent in Blacks and Hispanics. Neuroepidemiology. 1998;17:188-98.

7. Morgenstern LB, Smith MA, Lisabeth LD, Risser JMH, Uchino K, Garcia N, et al. Excess stroke in Mexican Americans compared with non-Hispanic Whites: the Brain Attack Surveillance in Corpus Christi Project. Am J Epidemiol. 2004;160:376-83.

8. Lisabeth LD, Smith MA, Brown DL, Moye LA, Risser JMH, Morgenstern LB. Ethnic differences in stroke recurrence. Ann Neurol. 2006;60:469-75.

9. Samsa GP, Bian J, Lipscomb J, Matchar DB. Epidemiology of recurrent cerebral infarction: a medicare claims-based comparison of first and recurrent strokes on 2-year survival and cost. Stroke. 1999;30:338-49.

10. Engel-Nitz NM, Sander SD, Harley C, Rey GG, Shah H. Costs and outcomes of noncardioembolic ischemic stroke in a managed care population. Vasc Health Risk Manag. 2010;6:905-13.

11. McGruder HF, Malarcher AM, Antoine TL, Greenlund KJ, Croft JB. Racial and ethnic disparities in cardiovascular risk factors among stroke survivors: United States 1999 to 2001. Stroke. 2004;35:1557-61.

12. Cushman M, Cantrell RA, McClure LA, Howard G, Prineas RJ, Moy CS, et al. Estimated 10-year stroke risk by region and race in the United States: geographic and racial differences in stroke risk. Ann Neurol. 2008;64:507-13.

13. Howard G, Lackland DT, Kleindorfer DO, Kissela BM, Moy CS, Judd SE, et al. Racial differences in the impact of elevated systolic blood pressure on stroke risk. JAMA Intern Med. 2013;173:46-51.

14. Ruland S, Richardson D, Hung E, Brorson JR, Cruz-Flores S, Felton WL, et al. Predictors of recurrent stroke in African Americans. Neurology. 2006:67:567-71.

15. Leoo T, Lindgren A, Petersson J, von Arbin M. Risk factors and treatment at recurrent stroke onset: results from the Recurrent Stroke Quality and Epidemiology (RESQUE) Study. Cerebrovasc Dis. 2008:25:254-60.

16. Friday G, Alter M, Lai S-M. Control of hypertension and risk of stroke recurrence. Stroke. 2002;33:2652-7.

17. Ozark S, Silver B. Secondary stroke prevention in 2011: an update on available options. Med Health R I. 2011:94:372-5.

18. Sacco RL, Adams R, Albers G, Alberts MJ, Benavente O, Furie K, et al. Guidelines for prevention of stroke in patients with ischemic stroke or transient ischemic attack: a statement for healthcare professionals from the
American Heart Association/American Stroke Association Council on Stroke: co-sponsored by the Council on Cardiovascular Radiology and Intervention: the American Academy of Neurology affirms the value of this guideline. Stroke. 2006:37:577-617.

19. Lawes CMM, Bennett DA, Feigin VL, Rodgers A. Blood pressure and stroke: an overview of published reviews. Stroke. 2004:35:1024.

20. Rashid P, Leonardi-Bee J, Bath P. Blood pressure reduction and secondary prevention of stroke and other vascular events: a systematic review. Stroke. 2003;34:2741-8.

21. Gordon NF, Gulanick M, Costa F, Fletcher G, Franklin BA, Roth EJ, et al. Physical activity and exercise recommendations for stroke survivors: an American Heart Association scientific statement from the Council on Clinical Cardiology, Subcommittee on Exercise, Cardiac Rehabilitation, and Prevention; the Council on Cardiovascular Nursing; the Council on Nutrition, Physical Activity, and Metabolism; and the Stroke Council. Stroke. 2004;35:1230-40.

22. Cruz-Flores S, Rabinstein A, Biller J, Elkind MSV, Griffith P, Gorelick PB, et al, Racial-ethnic disparities in stroke care: the American experience: a statement for healthcare professionals from the American Heart Association/American Stroke Association. Stroke. 2011;42:2091-116.

23. Caffrey C, Sengupta M, Moss A, Harris-Kojetin L, Valverde R. Home health care and discharged hospice care patients: United States, 2000 and 2007. Natl Health Stat Report. 2011:38:1-27.

24. Forster AJ, Murff HJ, Peterson JF, Gandhi TK, Bates DW. The incidence and severity of adverse events affecting patients after discharge from the hospital. Ann Intern Med. 2003;138:161-7.

25. Coleman EA, Boult C. Improving the quality of transitional care for persons with complex care needs. J Am Geriatr Soc. 2003;51:556-7.

26. Naylor MD, Brooten DA, Campbell RL, Maislin G, McCauley KM, Schwartz JS Transitional care of older adults hospitalized with heart failure: a randomized, controlled trial. J Am Geriatr Soc. 2004;52:675-84.

27. Brownstein JN, Chowdhury FM, Norris SL, Horsley T, Jack L, Zhang X, et al. Effectiveness of community health workers in the care of people with hypertension. Am J Prev Med. 2007;32:435-47.

28. Margolius D, Bodenheimer T, Bennett H, Wong J, Ngo V, Padilla G, et al. Health coaching to improve hypertension treatment in a low-income, minority population. Ann Fam Med. 2012;10:199-205.

29. Dye CJ, Williams JE, Evatt JH. Improving hypertension self-management with community health coaches. Health Promot Pract. 16 May 2014; EPub ahead of print.

30. Parry C, Coleman EA, Smith JD, Frank J, Kramer AM. The care transitions intervention: a patient-centered approach to ensuring effective transfers between sites of geriatric care. Home Health Care Serv Q. 2003:22:1-17.

31. Naylor MD, Bowles KH, McCauley KM, Maccoy MC, Maislin G, Pauly MV, et al. High-value transitional care: translation of research into practice. J Eval Clin Pract. 2013;19:727-33.

32. Naylor MD, Sochalski JA. Scaling up: bringing the transitional care model into the mainstream. Issue Brief (Commonw Fund). 2010;103:1-12.

33. Rosenthal EL, Wiggins N, Brownstein JN, Johnson S, Borbon IA, Rael R. The final report of the National Community Health Advisor Study. Tuscan, AZ: University of Arizona; 1998.

34. Bodenheimer TS. Training curriculum for health coaches. http://coloradoafp. org/library/Library\%20VI-Team\%20Care-1.pdf. Accessed: 5 February 2015.

35. Miller WR, Conforti SK. Motivational interviewing: preparing people for change. 2nd ed. New York: Guilford Publications, Inc; 2002.

36. Lane C, Huws-Thomas M, Hood K, Rollnick S, Edwards K, Robling M. Measuring adaptations of motivational interviewing: the development and validation of the behavior change counseling index (BECCI). Patient Educ Couns. 2005;56:166-73

37. Mahoney FI, Barthel DW. Functional evaluation: the Barthel Index. Md State Med J. 1965;14:61-5

38. EuroQol G. EuroQol - a new facility for the measurement of health-related quality of life. Health Pol. 1990;16:199-208

39. Diggle PJ, Liang K-Y, Zeger SL. Analysis of longitudinal data. Oxford: Oxford University Press; 1994.

40. Pragmatic, unifying algorithm gives power probabilities for common $F$ tests of the multivariate general linear hypothesis. http://www.bio.ri.ccf.org/ UnifyPow. Accessed: 5 February 2015.

41. Hardie K, Hankey GJ, Jamrozik K, Broadhurst RJ, Anderson C. Ten-year risk of first recurrent stroke and disability after first-ever stroke in the Perth Community Stroke Study. Stroke. 2004;35:731-5. 
42. Scmidt EV, Smirnov VE, Ryabova VS. Results of the seven-year prospective study of stroke patients. Stroke. 1988;19:942-9.

43. Center for Stroke Disparities Solutions. http://csds.med.nyu.edu. Accessed: 5 February 2015

44. Pavlik VN, Greisinger AJ, Pool J, Haidet P, Hyman DJ. Does reducing physician uncertainty improve hypertension control?: Rationale and methods. Circ Cardiovasc Qual Outcomes. 2009:2:257-63.

45. Bosworth HB, Powers BJ, Olsen MK, McCant F, Grubber J, Smith V, et al. Home blood pressure management and improved blood pressure control: results from a randomized controlled trial. Arch Intern Med. 2011;171:1173-80

46. Stewart S, Stocks NP, Burrell LM, de Looze FJ, Esterman A, Harris M, et al. More rigorous protocol adherence to intensive structured management improves blood pressure control in primary care: results from the Valsartan Intensified Primary carE Reduction of Blood Pressure study. J Hypertens. 2014;32:1342.

47. Ambrosius WT, Sink KM, Foy CG, Berlowitz DR, Cheung AK, Cushman WC, et al. The design and rationale of a multicenter clinical trial comparing two strategies for control of systolic blood pressure: the Systolic Blood Pressure Intervention Trial (SPRINT). Clin Trials. 2014;11:532-46.

48. Hebert PL, Sisk JE, Tuzzio L, Casabianca JM, Pogue VA, Wang JJ, et al. Nurse-led disease management for hypertension control in a diverse urban community: a randomized trial. J Gen Intern Med. 2012;27:630-9.

49. Bennett $\mathrm{H}$, Laird K, Margolius D, Ngo V , Thom DH, Bodenheimer T. The effectiveness of health coaching, home blood pressure monitoring, and home-titration in controlling hypertension among low-income patients: protocol for a randomized controlled trial. BMC Public Health. 2009;9:456.

50. Feldman PH, McDonald MV, Mongoven JM, Peng TR, Gerber LM, Pezzin LE. Home-based blood pressure interventions for blacks. Circ Cardiovasc Qual Outcomes. 2009;2:241-8.

51. James PA, Oparil S, Carter BL, Cushman WC, Dennison-Himmelfarb C, Handler J, et al. 2014 evidence-based guideline for the management of high blood pressure in adults: report from the panel members appointed to the Eighth Joint National Committee (JNC 8). JAMA. 2014;311:507-20.

52. Chobanian AV, Bakris GL, Black HR, Cushman WC, Green LA, Izzo JL, et al Seventh report of the Joint National Committee on Prevention, Detection, Evaluation, and Treatment of High Blood Pressure. Hypertension. 2003;42:1206-52.

53. Saver JL, Filip B, Hamilton S, Yanes A, Craig S, Cho M, et al. Improving the reliability of stroke disability grading in clinical trials and clinical practice: the Rankin Focused Assessment (RFA). Stroke. 2010;41:992-5.

54. Dubois B, Slachevsky A, Litvan I, Pillon B. The FAB: a Frontal Assessment Battery at bedside. Neurology. 2000;55:1621-6.

55. Charlson ME, Pompei P, Ales KL, Mackenzie CR. A new method of classifying prognostic comorbidity in longitudinal studies: development and validation. J Chronic Dis. 1987:40:373-83.

56. Chaudhry S, Jin L, Meltzer D. Use of a self-report-generated Charlson Comorbidity Index for predicting mortality. Med Care. 2005;43:607-15.

57. Rose M, Bjorner JB, Becker J, Fries JF, Ware JE. Evaluation of a preliminary physical function item bank supported the expected advantages of the Patient-Reported Outcomes Measurement Information System (PROMIS). J Clin Epidemiol. 2008;61:17-33.

58. Willey JZ, Williams O, Boden-Albala B. Stroke literacy in Central Harlem: a high-risk stroke population. Neurology. 2009;73:1950-6.

59. Morisky DE, Ang A, Krousel-Wood M, Ward HJ. Predictive validity of a medication adherence measure in an outpatient setting. J Clin Hypertens (Greenwich). 2008;10:348-54.

60. Horne RWJ, Hankins M. The Beliefs about Medicines Questionnaire: the development and evaluation of a new method for assessing the cognitive representation of medication. Psychol Health. 1999;14:1-24.

61. Glasgow RE, Wagner EH, Schaefer J, Mahoney LD, Reid RJ, Greene SM. Development and validation of the Patient Assessment of Chronic Illness Care (PACIC). Med Care. 2005:43:436-44.

62. Lorig K, Drph KLR, Stewart A, Ritter P, Gonzalez VM, Laurent D, et al. Outcome measures for health education and other health care interventions. Thousand Oaks, CA: Sage Publications; 1996.

63. Smith KW, Hoelscher DM, Lytle LA, Dwyer JT, Nicklas TA, Zive MM, et al. Reliability and validity of the Child and Adolescent Trial for Cardiovascular Health (CATCH) food checklist: a self-report instrument to measure fat and sodium intake by middle school students. J Am Diet Assoc. 2001;101:635-47.

64. Booth M. Assessment of physical activity: an international perspective. Res Q Exerc Sport. 2000;71:114-20.
65. Craig $C L$, Marshall AL, Sjostrom M, Bauman AE, Booth ML, Ainsworth BE, et al. International physical activity questionnaire: 12-country reliability and validity. Med Sci Sports Exerc. 2003;35:1381-95.

66. Pilkonis PA, Choi SW, Reise SP, Stover AM, Riley WT, Cella D. Item banks for measuring emotional distress from the Patient-Reported Outcomes Measurement Information System $\left(\mathrm{PROMIS}^{\circ}\right)$ : depression, anxiety, and anger. Assessment. 2011;18:263-83.

\section{Submit your next manuscript to BioMed Central and take full advantage of:}

- Convenient online submission

- Thorough peer review

- No space constraints or color figure charges

- Immediate publication on acceptance

- Inclusion in PubMed, CAS, Scopus and Google Scholar

- Research which is freely available for redistribution 